\title{
Improving Engineering Governance for Large Infrastructure Projects
}

\author{
William Scott', Gary Arabian², Peter Campbell ${ }^{1}$ and Richard Fullalove ${ }^{2}$ \\ ${ }^{1}$ SMART Infrastructure Facility, University of Wollongong, Australia \\ ${ }^{2}$ Asset Standards Authority, Transport for New South Wales, Australia
}

\begin{abstract}
The increased complexity of modern infrastructure projects together with the desire of governments to provide improved services to their citizens gives rise to the need for much better engineering governance capability and the ability to model system and user behaviour to ensure the desired increased level of service. Modelling all aspects of planned systems through the use of standardised architecture framework models that can be developed to provide the necessary insight across all aspects and levels of concern for the system(s) is an excellent approach for achieving this.

This paper describes the initial phase of development of such a toolset for the Asset Standards Authority (ASA) of Transport for New South Wales (TfNSW) that will be used to ensure coordinated development activities between all divisions in the department, as well as with Planning NSW and the private sector providers for system design, implementation, maintenance and operation. The initial development described here particularly focuses on existing, current and future heavy rail projects, but continuing work is under way to extend and generalise the model so that it applies to all rail transport modes (rapid transit metro and light rail) and eventually to include all other TfNSW transport modes - buses, walkways, ferries, cycle ways, and roads. Furthermore, this paper details how the use of a metamodel for the architecture framework provides the rigour and abstraction necessary to allow this generalisation within the same model structure.
\end{abstract}

Keywords: Enterprise Modelling, TRAK Meta-Model, SysML Modelling Language

\section{BACKGROUND}

The Australian State of New South Wales (NSW) has recently reorganised the way in which it will deliver new transportation assets (including infrastructure and vehicles). Existing capability has either been retained within the new Transport for NSW (TfNSW) organisation, or outsourced to the private sector. Six new divisions have been created within TfNSW with well-defined responsibilities for the delivery of an ambitious program of new transport assets for the state. At the same time it was recognised that this structure would need strong asset management governance to ensure that the new structure did not become siloed, thereby leading to asset planning and delivery mis-matches. In addition there is also the need to coordinate effectively with private sector providers as Authorised Engineering Organisations (AEOs). The means of achieving this has been to create the Assets Standards Authority (ASA).

"The Asset Standards Authority (ASA) is an independent unit established within Transport for NSW, and is the network design and standards authority for NSW transport assets. The ASA is responsible for developing engineering governance and frameworks to support industry delivery in assurance of design, safety, integrity, construction, commissioning, maintenance and disposal of transport assets for the whole asset life cycle. The ASA is also responsible for providing the standards for NSW rail assets, which industry organisations can use to deliver projects 
and manage assets in a more innovative, safe and efficient manner." (www.transport.nsw.gov.au/asa)

This presentation and paper describes architecture framework modelling that is being carried out by the ASA and the SMART Infrastructure facility at the University of Wollongong to provide the intellectual rigor and tools to accomplish these goals in a manner that is auditable, efficient and engages the stakeholders.

\section{APPROACH}

Delivering modern infrastructure is a complex enterprise involving many stakeholders namely; Politicians, government planning, Authorised Engineering Organisations (AEOs), design and acquisition agencies, finance organisations, private contractors, system operators and perhaps most important, the customers of TfNSW. The top level goal of TfNSW is the delivery of cost effective, reliable, sustainable, efficient, convenient and most importantly safe heavy rail and other services to the state's population to satisfy the expectations of the customers' experience.

The approach being considered and developed by ASA to deliver the structure to accomplish management of this Transport System of Systems (SOS) is the development of an architecture framework based toolset capable of describing and linking all key transport enterprise stakeholder concerns and involvements in the delivery process. The initial work described here has been the development of the toolset for the Heavy Rail sector of transportation, with the ultimate goal being to generalise the model that is the basis of the toolset to eventually include all transport modes in the framework, including multi-modal operations.

The top level concepts included in the toolset model development are being built using the TRAK metamodel and architecture framework (trak.sourceforge.net). The original TRAK metamodel and architecture framework was developed in the UK for application to railway projects, and was derived from the Ministry of Defence Architecture Framework (MODAF). The TRAK metamodel is now a general purpose and enterprise architecture framework which provides 5 key viewpoints into the enterprise, which in this case is taken as the provision of heavy commuter rail assets and services. These viewpoints are - Enterprise, Concept, Solution (Inclusive of solution resource), Procurement, and Management. The TRAK metamodel, being based on the Unified Modelling Language (UML) does not specify how these viewpoints are to be expressed as requirements and performance parameters. Therefore, in this work we have chosen to use SysML (www.sysml.org) because TfNSW requires understanding (and therefore representation) of low level functional behaviour of passengers, trains and elements of the railway system such as energy supply and train control, and SysML has the capacity to do this better than UML. Another need that TfNSW has is to be able to validate the requirements of its high level enterprise goals and capabilities in terms of traceability to the lower level functional and physical requirements that arise from them and SysML is able to support this through the use of its parametric diagram view. In addition to the modelling of the physical asset performance of the new heavy rail systems, this work is also developing the structure for modelling passenger behaviour throughout the use profile of passengers, and also specific scenarios such as the effects of passenger numbers on platform dwell times, and the effect these can have on the provision of high frequency train services.

\section{MODEL DEVELOPMENT TO MID 2014}

In this section we illustrate with a few of the model elements the manner in which an Architecture framework model can depict and link all levels of an enterprise, so that knowledge about the system at all stages can be accessed in the context of the system as a whole. While this can be seen as a lot of work - and it certainly is - all evidence is that the ROI can be huge in the avoided cost of later scope and performance errors and shortfalls being discovered at a stage in the project where correcting them is hugely expensive and usually leads to significant delays.

At the enterprise level the enterprise goals (as stated in the NSW Long Term Transport Master Plan) required a set of supporting capabilities as shown in Figure 1. The Concept Activities that support these capabilities where then identified as shown in Figure 2. This diagram captures the traceability of which concept activities directly support the required capabilities. In this way the diagram both provides justification for these needed activities and provides the structure and location in the model where evidence that all capabilities have been addressed can be either confirmed, or if absent, cause early corrective action to be taken. 

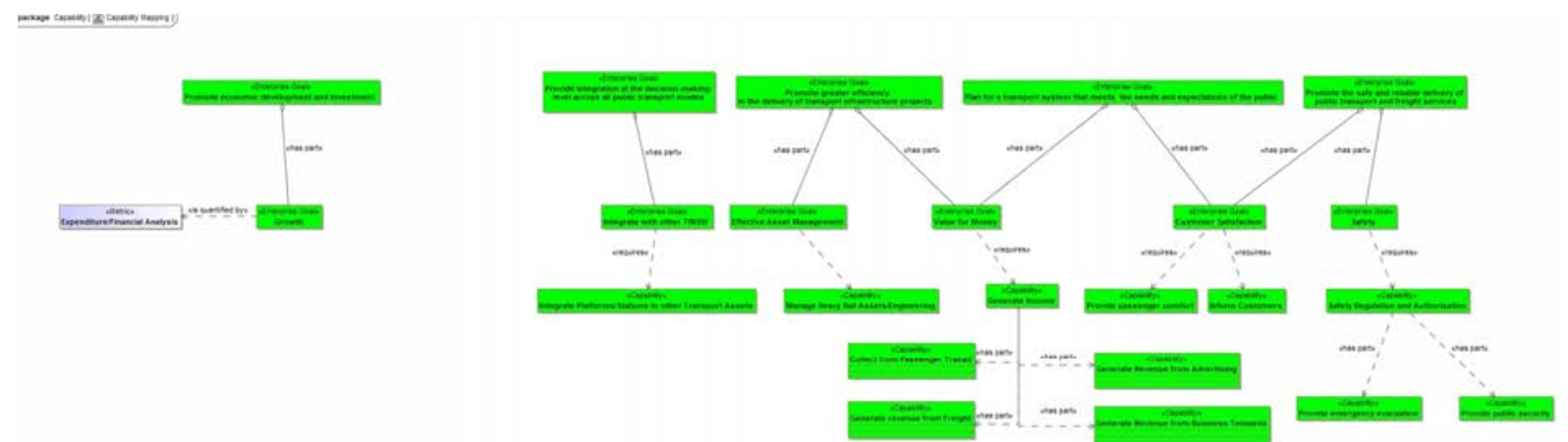

Figure 1: Capability mapping based on enterprises goals

Although some of these diagrams (such as Figures 1 and 6) are often impossible to read on the page of a document this is not an issue in using the model as they are designed as entry points into the model and its database rather than only being guides to enterprise structure. The model is designed and expected to only be used on a capable computer system where such diagrams can always be expanded so that the diagrams can be read.

bdd [Package] Capability [ CVp-04 Concept Activity to Capability Mapping - Rolling Stock 1]

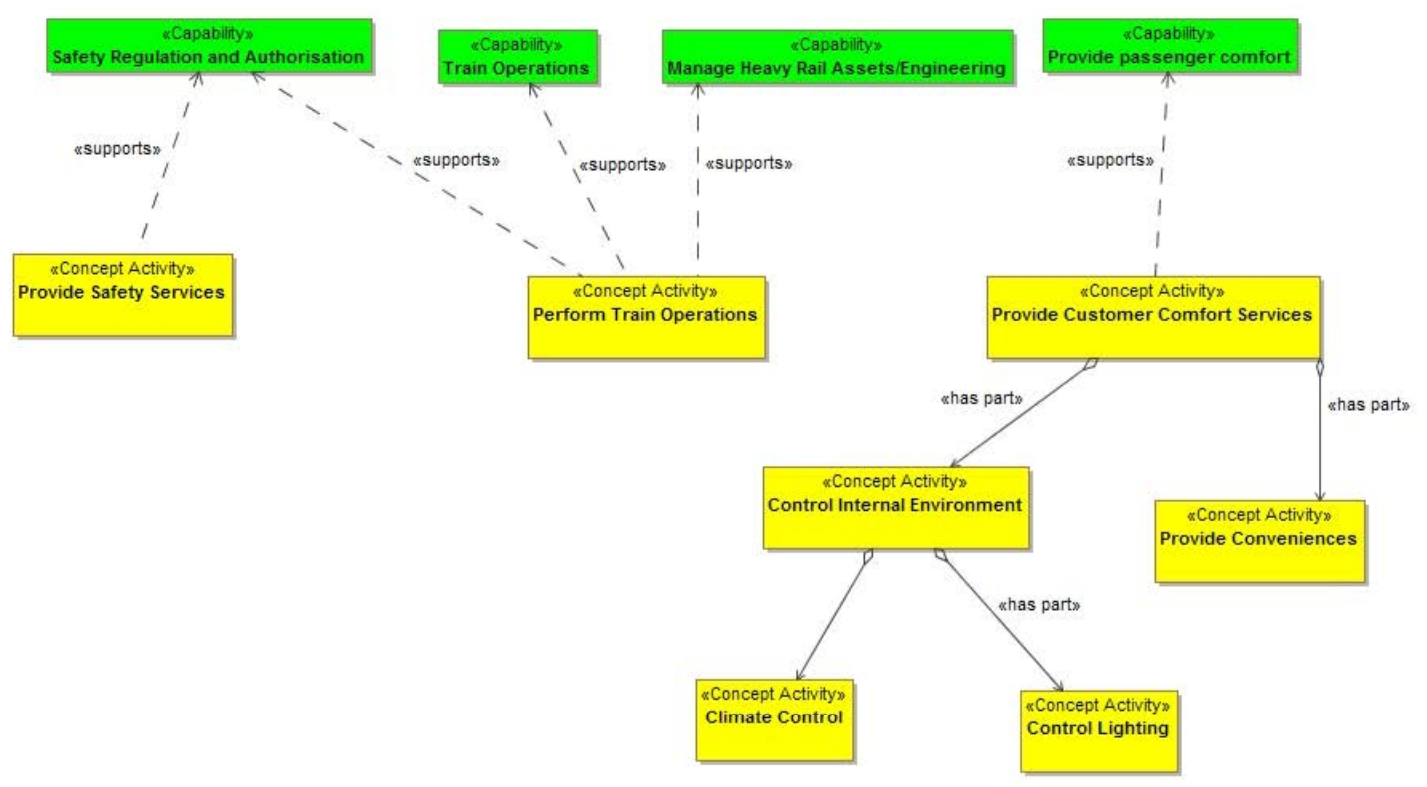

Figure 2: Capabilities mapped onto concept activities

The initial model developed by ASA using TRAK, on which this SysML model is based, defines the major system elements as rolling stock, stations and buildings, telecommunications, electrical supply, signals, rail network and management. Development so far has focused on the rolling stock, telecommunications and stations and buildings, though aspects of the other elements have been defined where they touch upon the model behaviour. Here we describe the development of the stations and buildings component to illustrate the development process at a lower level.

The station operations conceptual hierarchy model is shown in Figure 3. 


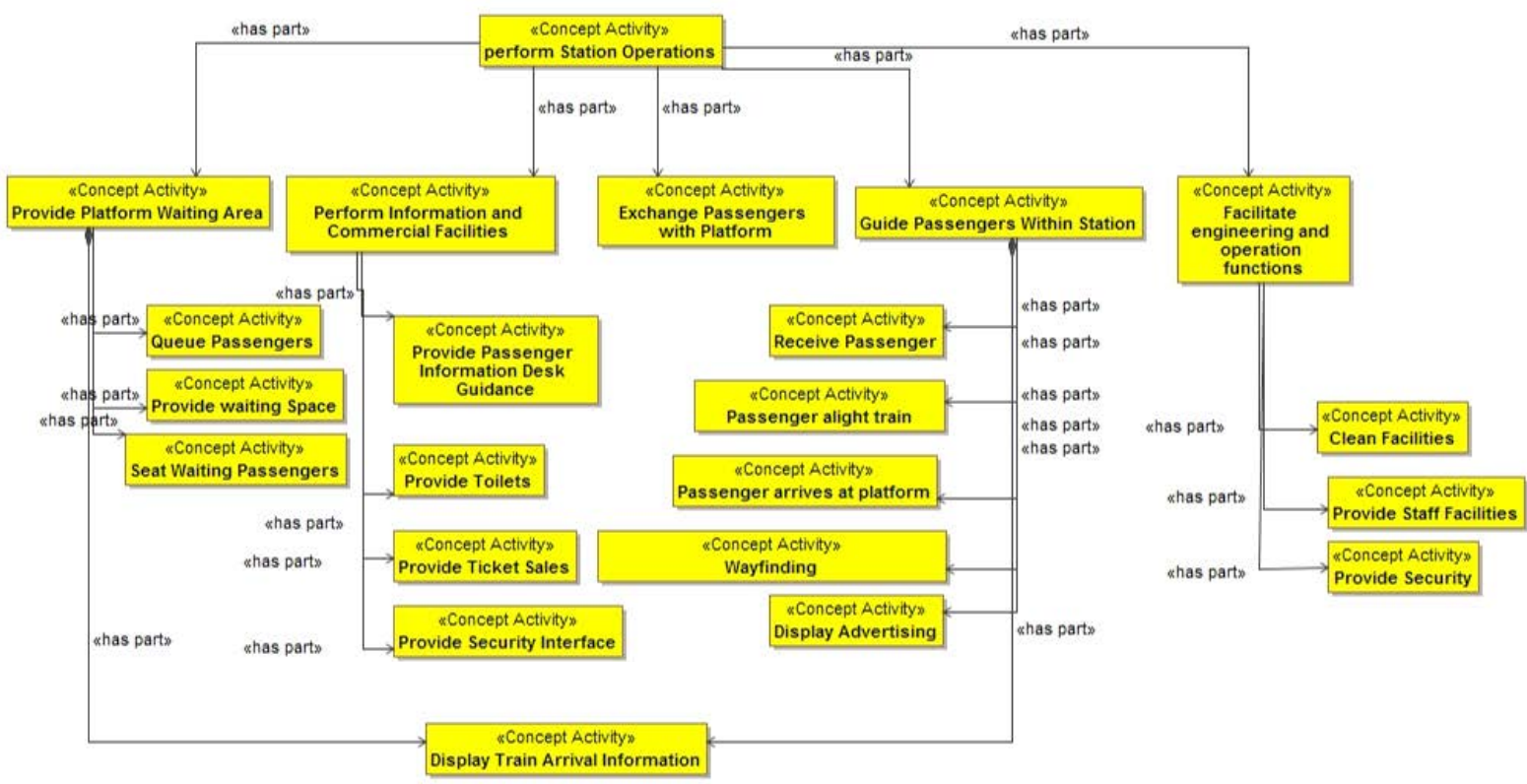

Figure 3: Station operations conceptual activity hierarchy

The diagram shows a representative sample collection of concept activities that are needed to perform the necessary station operations of the rail system, and the dependencies between them. Each of the nodes (logical operational entities) in the diagram can then be further developed with more detail, including the standards that apply and eventually the design documents and validation activities.

Figure 4 shows an example of the concept activities that are needed to guide passenger within a station. Note that this is still done at an abstract level, so that the diagram has general applicability. Here it applies to a railway station, but a very similar diagram would also apply to passengers using a ferry service - where the platform becomes a wharf, but otherwise activities are similar or the same at the abstract level, and then can be instantiated with the particular details for each application.

At a lower level still, Figure 5 is a parametric diagram showing the model structure into which the behaviour models in this case either a simple queuing model or possibly an agent based model capable of modelling passengers with individual attributes - can be executed to validate the capability of optional numbers of gates to meet the expected passenger use rates. 


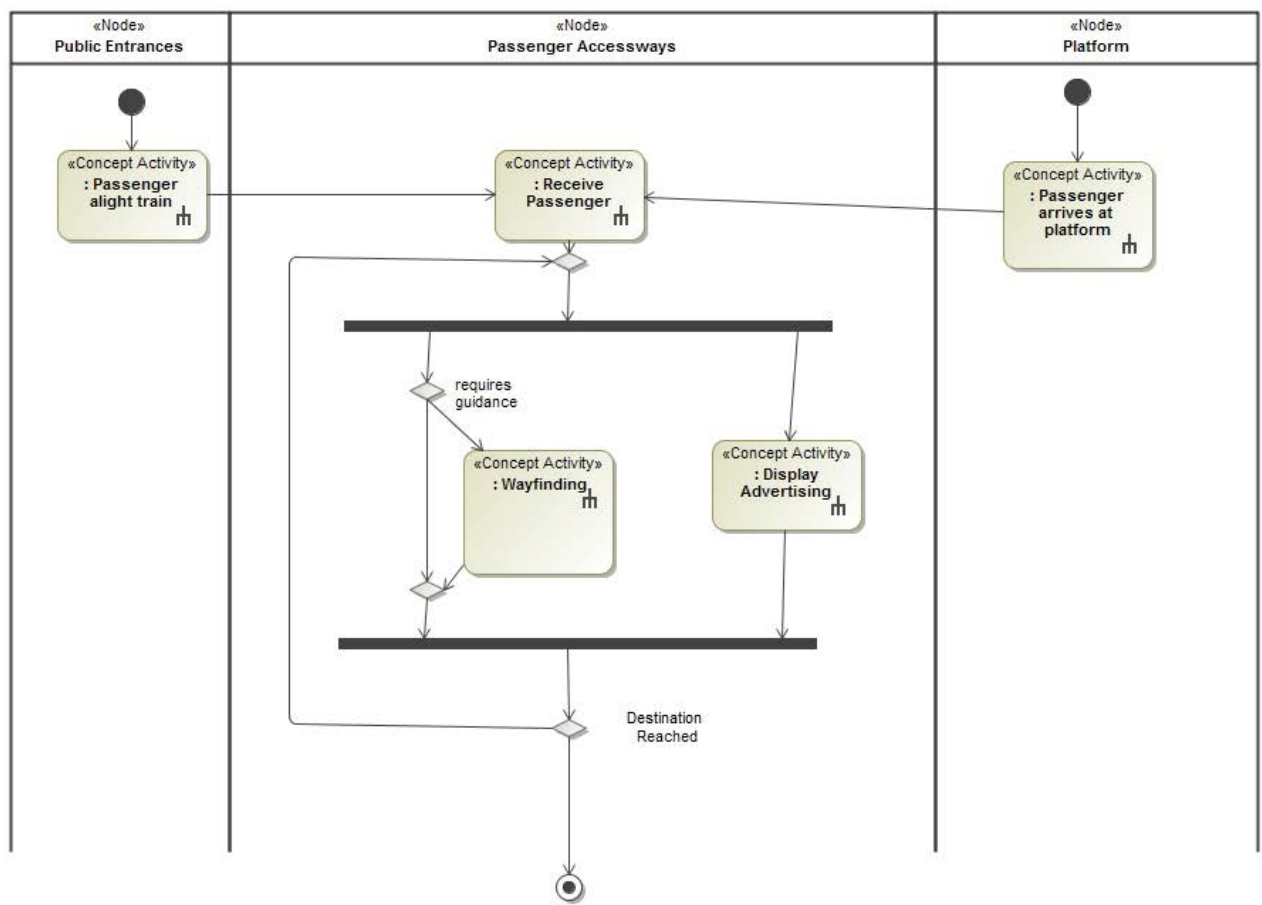

Figure 4: Interaction between concept activities.

par [Class] Revenue Protection Barrier [ Revenue Protection Barrier ]]

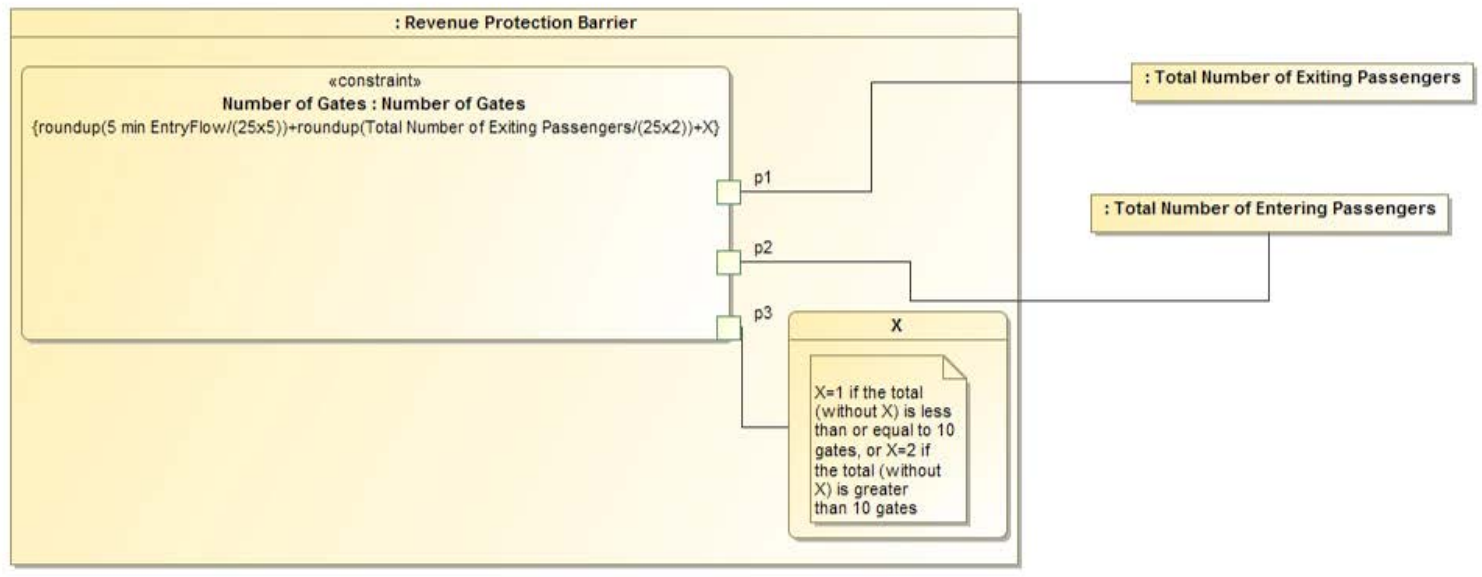

Figure 5: Parametric diagram to determine the number of gates needed in a revenue protection barrier

As embodied in its title, the ASA is particularly concerned with improving the ability of TfNSW to manage the impact of standards on the development of future assets. To date it has identified over 1,000 standards that potentially apply to its current heavy rail assets - which includes a new high frequency line to the North West suburbs of Sydney and upgrades to a higher level of service on the main western line from the CBD through to North Sydney. In addition to this large body of documents, which contains figures and diagrams as well as text, any asset type also has the specific documents developed for its design and realisation, and sitting above all this are the various acts and other high level legal instruments under which TfNSW must function. One of the goals that TfNSW and ASA have is to move the existing approach based on specify type standards to functional and performance based standards, with the expectation that this will allow more scope for innovations by the supply chain in procuring future transportation assets. In carrying out this objective, the ability to understand how the multitudes of standards are linked to all the asset design elements is of extreme importance.

The tools available in the model also provide the opportunity for linking documents directly to the database.

Stakeholders need to specify applicable documents and standards that have to be met, or guidance that influences 
the design of the systems. These documents can often specify further documentation to cause a traceability chain that, if unmanaged, can cause difficulties in maintaining adequate knowledge of the system as a whole. Standards and legislation can change, so that the new standard needs to be re-examined to determine the implications of these changes. There have also been cases where cyclic references have been generated by standards produced in isolation. The way in which the model has been developed to address the need for document traceability is shown in Figure 6.
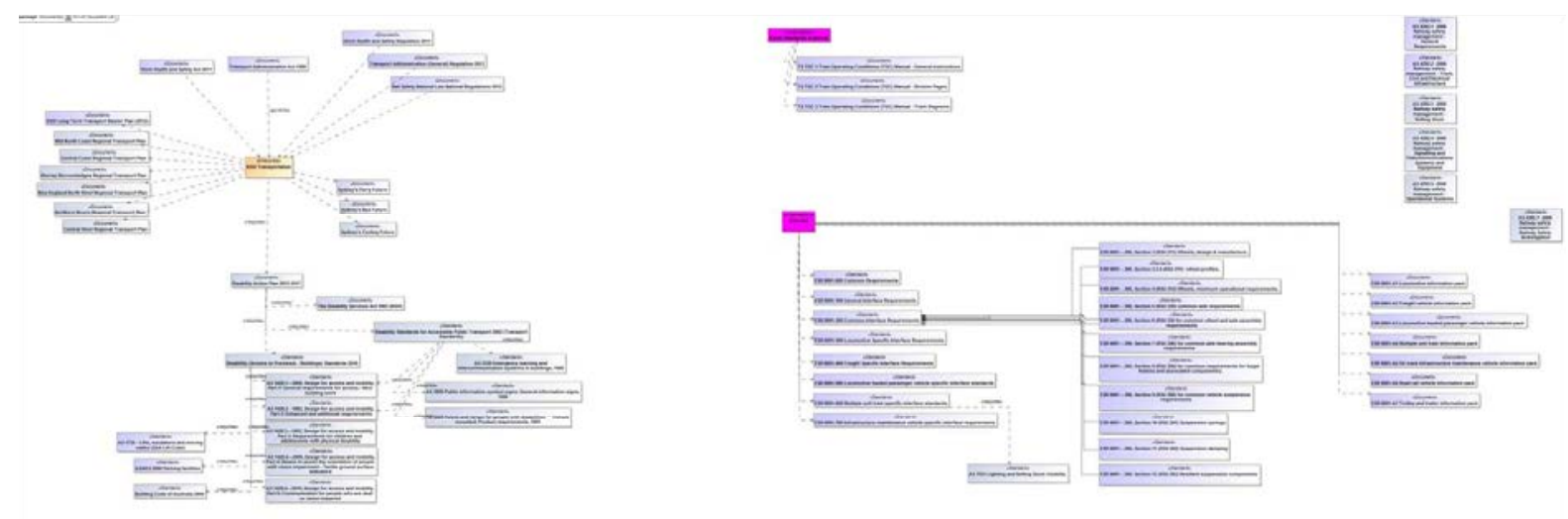

\section{Figure 6: Document library}

The documents shown in the library diagram can be linked either to copies found either on the same machine as the model or via a URL. This helps maintain the connectivity of the model information as the "single source of truth" with relevant documentation and makes document navigation easier. The application of the SysML model to the management of standards can be illustrated by the following example of the standards that apply to detailed physical design level for a carriage bogie. Figure 7 shows the way in which the nodes in a bogie design can be traced through the requirements back to the applicable standards.

\section{CONCLUSION}

As illustrated by the set of diagrams presented here, the use of an architecture framework type model expressed in SysML provides a powerful toolset for managing the engineering and behavioural aspects of large complex transport systems. At the top level many stakeholders and their expectations are modelled so that the interdependencies can be managed in a timely manner. At the lowest level the hundreds of thousands of standards, design documents, requirements specifications can all be placed in a common data base and linked to the applicable nodes and viewpoints in the model. As the complexity of modern transport projects continues to increase through the need for provision for greater efficiency, improved safety and greater user satisfaction, the need for such a model based systems engineering approach and toolset is becoming more and more urgent. A major issue in developing such tools is the dearth of trained people with the ability to do so effectively.

What has been described here is a work in progress. In addition to continuing the present development to support more detailed toolset capability, it is planned to extend the model to a generalised version that can be used for all transport modes in NSW, and to ensure that the requirements diagrams in the model are structured to support the automatic generation and export of text requirements in the nearly ubiquitous requirements tool DOORS that is used elsewhere in TfNSW. Research will also be conducted into the feasibility of machine reading of the requirements arising from the standards as an aid to coping with the huge load involved in applying them to new work as well as the transforming them to a performance based form. 


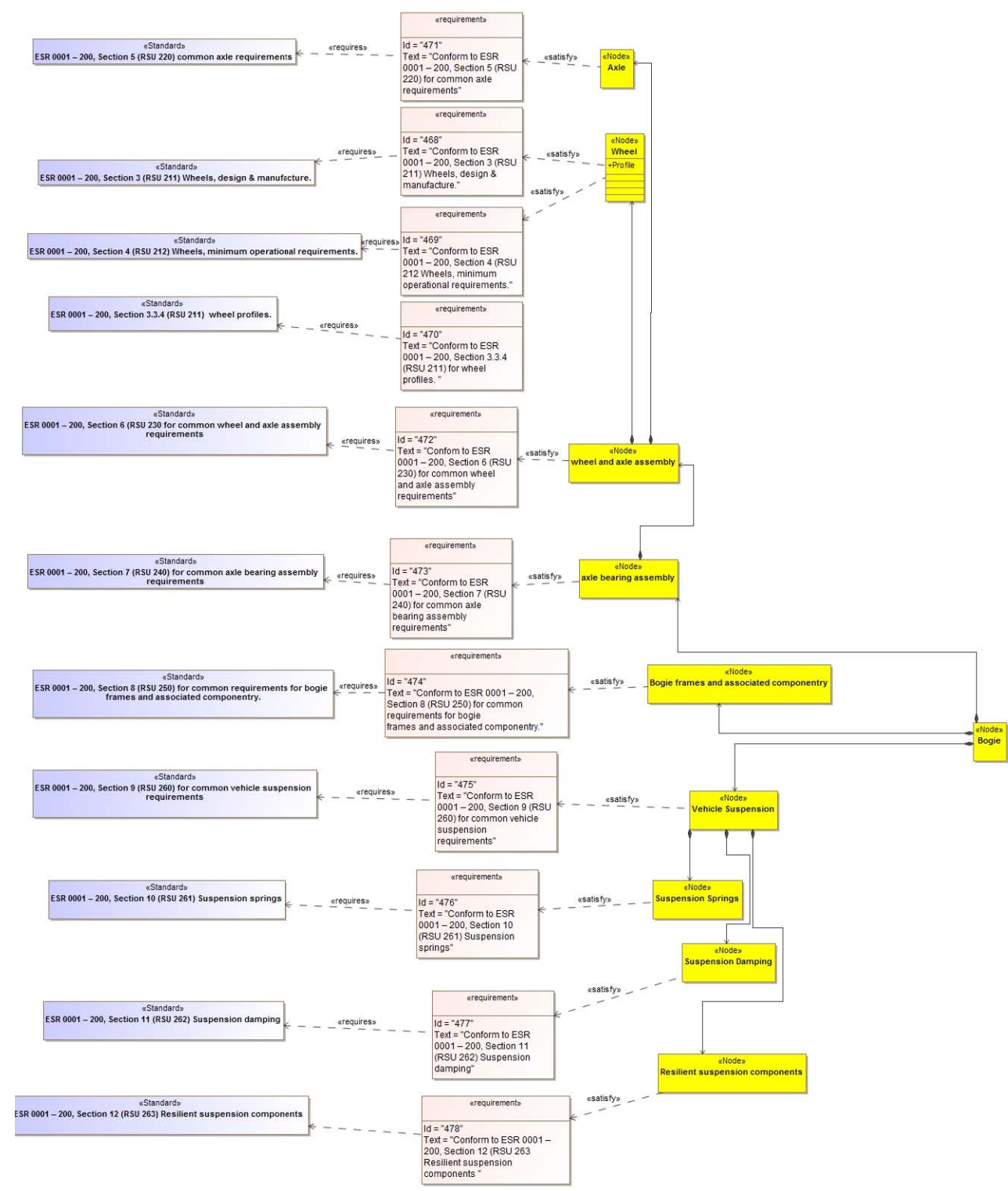

Figure 7: Traceability from nodes through requirements to stand 